Gut and Liver, Vol. 10, No. 5, September 2016, pp. 663-664

\title{
The Clinical Utility of MicroRNA as a Prognostic Biomarker of Pancreatobiliary Cancers
}

\author{
Kyu Taek Lee \\ Department of Medicine, Samsung Medical Center, Sungkyunkwan University School of Medicine, Seoul, Korea
}

See "MicroRNA 141 Expression Is a Potential Prognostic Marker of Biliary Tract Cancers" by Jaihwan Kim, et al. on page 836, Vol. 10. No. 5, 2016

A microRNA (miRNA) is a small noncoding RNA molecule (containing about 22 nucleotides) found in plants, animals and some viruses that functions in RNA silencing and posttranscriptional regulation of gene expression. The miRNA induces gene silencing by binding to the 3'-untranslated regions (3'-UTRs) of target mRNAs. This interaction prevents protein production by inhibition of protein translation and/or by mRNA degradation. Therefore, miRNAs have been reported to have important functions in the regulation of carcinogenesis and cancer progression as well as homeostasis. This explains why miRNAs are intensely studied as candidates for diagnostic and prognostic biomarkers in cancers.

The miRNAs are invariably found to be misexpressed in every type of cancer examined so far, and miRNA genes can act as both tumor-suppressor genes and oncogenes. There were many studies about miRNA in pancreatobiliary cancers. Ali et al. ${ }^{1}$ suggested that serum miR-21 and other miRNAs could predict the aggressiveness of pancreatic cancer. This miR-21 is overexpressed in cholangiocarcinoma and the knockdown of miR-21 resulted in inhibition of cellular invasion and metastasis. ${ }^{2}$ As a result, miR-21 may be a potential biomarker for pancreatobiliary cancer prognosis. A combination of serum eight miRNAs (miR-6075, miR-4294, miR-6880-5p, miR-6799-5p, miR-125a3p, miR-4530, miR-6836-3p, and miR-4476) suggested as early diagnostic biomarkers to detect pancreatobiliary cancers. ${ }^{3}$ The sensitivity, specificity, and accuracy to detect pancreatobiliary cancers showed superiority comparing to carbohydrate antigen 19-9 and carcinoembryonic antigen.

Pancreatobiliary cancers are highly malignant tumors and the overall prognosis is poor. This is why the new prognostic mark- ers for pancreatobiliary cancers are very important and essential to treat and follow up on the aggressive cancers. In this issue of Gut and Liver, Kim et al. ${ }^{4}$ suggested that miRNA 141 may be a valuable prognostic biomarker in patients with biliary tract cancer. The miRNA 141 highly overexpressed in malignant cholangiocytes was suggested as an important regulator of tumor cell proliferation in vitro and decreasing miRNA 141 overexpression may be useful to inhibit tumor progress in previous study. ${ }^{5} \mathrm{Au}-$ thors demonstrated that this miRNA 141 overexpression was related to poor disease-free survival in patients with biliary tract cancers. Moreover, they elucidated that miRNA 141 overexpression was significantly associated with angiolymphatic invasions by using surgical specimens. This study has an important clinical impact to clarify the function of miRNA 141 in carcinogenesis of biliary tract cancers. Nevertheless, there are several limitations in this study. Authors investigated only the tissue sample but not the serum sample. It is very important to know the result of serum sample since it increases clinical utility due to its easy sampling and continuous monitoring with the follow up. The number of patients which showed high expression of miRNA 141 was only 18\%. Consequently, this alone cannot predict the poor prognosis of biliary tract cancers. Hence, for this reason other miRNAs or biomarkers should be combined to predict accurate prognosis. Recently, serum miR-26a was suggested as a potential prognostic biomarker in patients with cholangiocarcinoma. $^{6}$

MicroRNAs have important roles in cancer by targeting corresponding mRNAs and by influencing hallmarks of cancer. This is related to cell proliferation, avoiding growth suppression, activating invasion and metastasis, angiogenesis, and resisting

Correspondence to: Kyu Taek Lee

Department of Medicine, Samsung Medical Center, Sungkyunkwan University School of Medicine, 81 Irwon-ro, Gangnam-gu, Seoul 06351, Korea

Tel: +82-2-3410-3406, Fax: +82-2-3410-6983, E-mail: ktcool.lee@samsung.com

pISSN 1976-2283 eISSN 2005-1212 http://dx.doi.org/10.5009/gnl16309

@) This is an Open Access article distributed under the terms of the Creative Commons Attribution Non-Commercial License (http://creativecommons.org/licenses/by-nc/4.0) which permits unrestricted non-commercial use, distribution, and reproduction in any medium, provided the original work is properly cited. 
cell death. As a result, finding new prognostic biomarkers of microRNAs provide essential knowledge for prediction of patient prognosis and for discovery of new therapeutic targets for cancer treatment.

\section{CONFLICTS OF INTEREST}

No potential conflict of interest relevant to this article was reported.

\section{REFERENCES}

1. Ali S, Almhanna K, Chen W, Philip PA, Sarkar FH. Differentially expressed miRNAs in the plasma may provide a molecular signature for aggressive pancreatic cancer. Am J Transl Res 2010;3:2847.
2. Huang Q, Liu L, Liu CH, et al. MicroRNA-21 regulates the invasion and metastasis in cholangiocarcinoma and may be a potential biomarker for cancer prognosis. Asian Pac J Cancer Prev 2013;14:829-834.

3. Kojima M, Sudo H, Kawauchi J, et al. MicroRNA markers for the diagnosis of pancreatic and biliary-tract cancers. PLoS One 2015;10:e0118220.

4. Kim J, Ryu JK, Lee SH, Kim YT. MicroRNA 141 expression is a potential prognostic marker of biliary tract cancers. Gut Liver 2016;10:836-841.

5. Meng F, Henson R, Lang M, et al. Involvement of human microRNA in growth and response to chemotherapy in human cholangiocarcinoma cell lines. Gastroenterology 2006;130:2113-2129.

6. Wang LJ, Zhang KL, Zhang N, et al. Serum miR-26a as a diagnostic and prognostic biomarker in cholangiocarcinoma. Oncotarget 2015;6:18631-18640. 